\title{
Performance Evaluation of Log-normal And Negative Exponential Channel Modeling Using Various Modulation Techniques in OFDM-FSO Communication
}

\author{
Nitin Shankar Singh, Gurpartap Singh \\ Department of electronics and communication, Lovely Professional University, Phagwara, India \\ nitin.shankarsingh@gmail.com \\ Assistant Professor, Lovely Professional University, Phagwara, India
}

gurpartap.15882@|pu.co.in

\begin{abstract}
Free Space Optics (FSO) is one of the emerging technology which is thoroughly being popular and is basically using optical signals for the communication. The paper evaluates the bit error rate performance of the Free space Optics (FSO) system.To design a high performance communication link for the atmospheric FSO channel, it is of great importance to characterize the channel with proper model. The performance of the highly efficient, high data rate system is limited by the certain constraints like scintillation effects and atmospheric turbulence. The FSO communication system using Orthogonal Frequency Division Multiplexing (OFDM) technique which is known for its increased robustness against frequency selective fading, narrow-band interference and high channel efficiency. The performance of the modulation techniquesBinary Phase Shift Keying (BPSK), Quadrature Phase Shift Keying (QPSK) and 8-PSK are studied in the Lognormal and Negative Exponential channel. The obtained results are the comparative study of Bit Error Rate (BER) vs. Signal to Noise Ratio (SNR), which is quite useful for analyse the system performance with different channel models for various modulation techniques.
\end{abstract}

\section{General Terms}

Atmospheric Turbulence, Bit Error Rate, Probability Density Function, Scintillation effects, Signal to Noise Ratio

Indexing terms

BER, BPSK, OFDM, PDF, QPSK, SNR, FSO, 8-PSK

\section{Academic Discipline And Sub-Disciplines}

This paper belongs to Electronics and communication academic disciplines.

\section{SUBJECT CLASSIFICATION}

Optical Communication; Free space Optical communication.

\section{COVERAGE}

This paper covers the free space optical communication system analysis over BER vs. SNR parameters for log normal and negative exponential channel modeling through various modulation techniques.

\section{TYPE (METHOD/APPROACH)}

The performance of different channel models has been studied and then implemented to a new system which is OFDMFSO with different modulation techniques (i.e. BPSK, QPSK and 8-PSK). the evaluation of these modulating techniques with Log-normal distribution and Negative Exponential distribution is compared. This new phenomenon gives better result than the previous techniques used.

\section{INTRODUCTION}

The Free Space Optics (FSO) systems are increasingly being considered as a suitable alternative technology for optical fiber networks, especially in areas where the deployment of optical fiber is not feasible and in underserved rural areas lacking broadband network connectivity. The advantages of FSO communications, depending on deployment scenario and application, including ease of deployment, license-free operation, high transmission security, high bit rates, full duplex transmission and protocol transparency, Free space optics (FSO) communications gives user a large and unregulated bandwidth. The free space optical system uses the Optical signal which carries the information. This optical signal is not confined into a physical channel like Optical Fiber. In the Free Space Optical communication the optical signal is transmitted into the free space and the air or vacuum space acts as the channel for signal transmission. The FSO can provide data rate in the range of $100 \mathrm{Gbit} / \mathrm{s}$ and the data transfer is achievable over a distance of $1-4 \mathrm{~km}$. The direct lineof-sight FSO link offer numerous advantages compared to the conventional wired and wireless communications [1]. 
Free space optical (FSO) communications is a promising technology capable of offering full-duplex gigabit rate throughput (data, voice, and video simultaneously) in certain applications and environment [2, 3]. FSO offers a huge license-free frequency spectrum using a single wavelength, immunity to electromagnetic interference including co/adjacent channel interference (due to a well-defined narrow beam size and no power spill), and high security [4]. FSO is less affected by snow and rain, but can be severely affected by the atmospheric turbulence and fog. The earth's atmosphere has three main hurdles to overcome when using it as a communication channel; absorption, scattering, and turbulence. Absorption of optical waves results in attenuation, it occurs throughout the visible and IR spectrum. Absorption is a selective process and results from specific molecules in the atmosphere having an absorption band at an optical wavelength. Scattering occurs when a particle in the atmosphere is on the same order of magnitude of the optical wavelength $[5,6]$.

The interaction of the particle and light wave causes an angular redistribution of a portion of the radiated wave. Optical turbulence is a result of fluctuations in the index of refraction along a propagation path. These fluctuations distort the phase front and vary the temporal intensity of an optical wave. The combination of these atmospheric effects on an optical system can cause phenomena such as beam spreading, image dancing, beam wander, and scintillation [7]. The dominant noise source in an FSO communication system is atmosphere-induced intensity and phase fluctuations; scintillation. A larger aperture (collecting lens) can help reduce scintillation effects and improve SNR. Design criteria such as detector threshold level, probability of detection, mean fade time, number of fades, and SNR require knowledge of the probability density function (PDF) of the received irradiance of the optical field. Orthogonal Frequency Division multiplexing (OFDM) is a popular modulation/multiplexing technique for broadband wireless communication which is robust to multipath fading and frequency selective fading. By this virtue, OFDM has become a modulation technique for IEEE 802.11a Wireless Local Area Network and IEEE 802.16 standards. Combining OFDM with FSO gives rise to OFDM based FSO which will exploit the advantages of both becomes a good candidate for "last mile" solution for broad band connectivity with high speed data rates [8].

\section{SYSTEM MODEL}

OFDM-FSO system support high data rates by splitting a high-rate data-stream into a number of low-rate data-streams and transmitting these over a number of narrowband subcarriers. Where OFDM is a kind of multicarrier transmission in which high data rate streams are split into lower rate streams and then transmitted simultaneously over several narrowband subcarriers. The subcarriers are themselves modulated by using phase shift keying (PSK) or quadrature amplitude modulation (QAM) and are then carried on a high frequency carrier. OFDM can be simply defined as a form of multicarrier modulation where its carrier spacing is carefully selected so that each subcarrier is orthogonal to the other subcarriers and can be separated at the receiver by correlation techniques; hence, intersymbol interference among channels can be eliminated. The set of orthogonal carriers is realized by using the inverse fast Fourier transform (IFFT) at the transmitter and the fast Fourier transform (FFT) at the receiver and requires no equalization [9]. The OFDM signal for $\mathrm{N}$ subcarriers, after up-conversion to the wireless service carrier frequency $f_{c}$, can be written as:

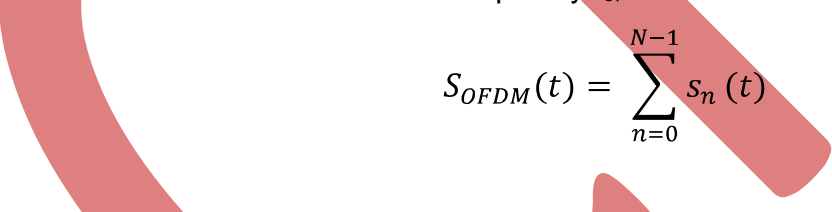

The first raw data is mapped according to different types of modulation techniques (BPSK, QPSK, 8-PSK, 16QAM, 64QAM), depending upon data rate. Representing from the above equation in complex data symbol, we have:

$$
S_{\text {OFDM }}(t)=\sum_{n=0}^{N-1} X_{n} \exp \left(j\left(\omega_{n}+2 \pi f_{c}\right) t\right), \quad 0<t<T s
$$

The above equation represents each symbol $X_{n}$ is amplitude modulated on orthogonal subcarriers. This process is performed using the IFFT which guarantees that all the subcarriers are orthogonal to each other over the symbol interval. Here, we set the guard interval to zero and thus the OFDM symbol duration Ts equals to the Fourier analysis window. The $\mathrm{SOFDM}_{(\mathrm{t})}$ is real by enforcing the conjugate-symmetry (Hermitian symmetry) of the IFFT input vector, The first input $\mathrm{X}_{0}$, corresponding to the zero frequency, needs to be real-valued and is generally left unmodulated. This approach with realvalued IFFT output is used in digital subscriber line (DSL) systems and is known as Discrete Multitone (DMT). Due to frequency selectivity, the subcarriers experience in general different channel gains which can be mitigated through the use of many narrow subcarriers. The signal SOFDM $(t)$ is then used to modulate the optical intensity of laser diode (LD) to be transmitted through fiber optics $[9,10]$. The basic configuration of OFDM FSO signal transmission over FSO link is shown in Fig. 1: 


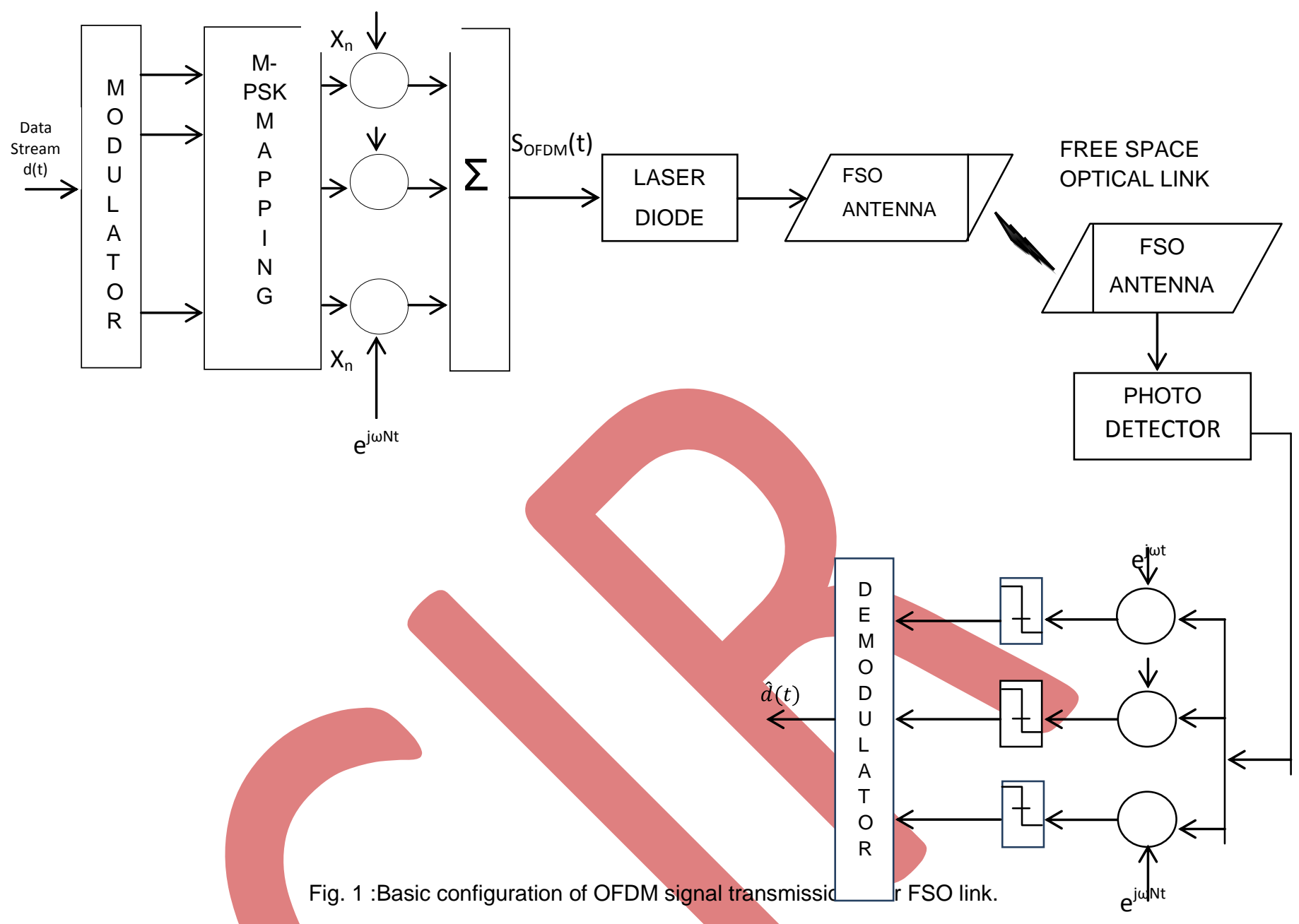

\section{Channel Modeling}

A commonly used turbulence model assumes that the variations of the medium can be understood as individual cells of air or eddies of different diameters and refractive indices. In the context of geometrical optics, these eddies may be thought of as lenses that randomly refract the optical wave front, producing a distorted intensity profile at the receiver of acommunication system. The most widely accepted theory of turbulence is due to Kolmogorov [11]. This theory assumes that kinetic energy from large turbulent eddies, characterized by the outer scale $L_{0}$, is transferred without loss to eddies of decreasing size down to sizes of a few millimetres characterized by the inner scale $I_{0}$. The inner scale represents the cell size at which energy is dissipated by viscosity. The refractive index varies randomly across the different turbulent eddies and causes phase and amplitude variations to the wave front. Turbulence can also cause the random drifts of optical beams a phenomenon usually referred to as wandering and can induce beam focusing. The reliability of the communication link can be determined if we use a good probabilistic model for the turbulence. To design a highperformance communication link for the atmospheric free-space optical (FSO) channel, it is of great Importance to characterize the channel. Several probability density functions (PDFs) have been proposed for the intensity variations at the receiver of an optical link. The atmospheric turbulence impairs the performance of an FSO link by causing the received optical signal to vary randomly thus giving rise to signal fading. The fading strength depends on the link length, the wavelength of the optical radiation and the refractive index structure parameter $C n^{2}$ of the channel. This model is mathematically tractable and it is characterized by the Rytov variance $\sigma_{R}{ }^{2}$. The turbulence induced fading is termed weak when $\sigma_{R}{ }^{2}<1$ and this defines the limit of validity of the model.

$$
\sigma_{R}^{2}=1.23 C_{n}^{2} k^{7 / 6} L^{11 / 6}
$$

$k=2 \pi / \lambda$ is the optical wave number, $L$ is propagation distance, and $C_{n}{ }^{2}$ is the refractive indexstructure parameter, which 4 we assume to be constant for horizontal paths.

\section{Log Normal Distribution}

A continuous distribution in which the logarithm of a variable has a normal distribution. A log normal distribution results if the variable is the product of a large number of independent, identically-distributed variables in the same way that a normal distribution results if the variable is the sum of a large number of independent, identically-distributed variables. 
The log-normal distribution model is consider for the weak regime, assumes the log intensity 'l'of the optical beam traversing the turbulent atmosphere to be normally distributed with a mean value of $-\sigma^{2} / 2$. Thus the probability density function of the received irradiance is given by[12]:

$$
p(I)=\frac{1}{\sqrt{2 \pi \sigma_{l}^{2}}} \frac{1}{I} \exp \left\{-\frac{\left(\ln \frac{I}{I_{0}}\right)-E[I]^{2}}{2 \sigma_{l}^{2}}\right\} \quad I \geq 0
$$

Where, Irepresents the irradiance at the receiver and $I_{0}$ is the signal irradiance without scintillation.

\section{Negative Exponential Model}

In the limit of strong irradiance fluctuations (i.e. in saturation regime and beyond) where thelink length spans several kilometres, the number of independent scatterings becomes large. This saturation regime is also called the fully developed speckle regime. The amplitude fluctuation of the field traversing the turbulent medium in this situation is generally believed and experimentally verified to obey the Rayleigh distribution implying negative exponentialstatistics for the irradiance. That is[13]:

$$
\boldsymbol{p}(I)=\frac{I}{I_{0}} \exp \left(-\frac{I}{I_{0}}\right) \quad I_{0}>0
$$

Where, $E[I]=I_{0}$ is the mean received irradiance. During the saturation regime, the value of the scintillation index, $S . I .=1$.

\section{DEPICTION OF FREE SPACE SNR}

The performance of a receiver is often based on the notion of signal-to-noise ratio SNR, i.e., the rms signal power over therms noise power. For photon-noise limited performance, the mean SEPfora direct detection system is $[14,15]$ :

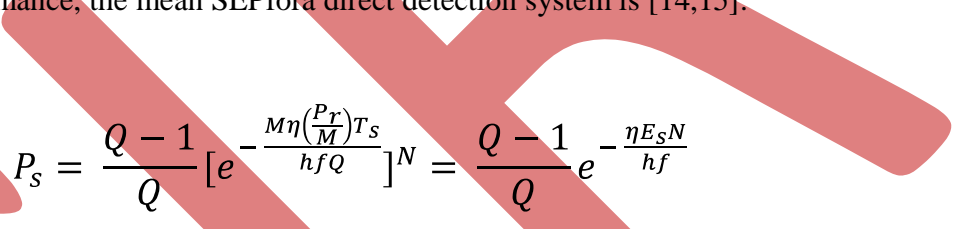

If the path gains are independently distributed and identical, the average symbol error is given by:

$$
P_{s}=\frac{Q-1}{Q}\left\{\left[\int e^{\frac{\alpha^{2} \eta\left(\frac{E_{S}}{M}\right)}{h f}} f(I) d I\right]^{N}\right\}^{M}
$$

System performances for continuous wave (CW) or single pulse optical systems are based on SNR and fade probability. For digital transmission of information, the performance measure is based on the probability of error, also called the bit error rate BER.

\section{RESULTS AND DISCUSSION}

The two distribution models discussed here with different modulation techniques (i.e. BPSK, QPSK and 8-PSK) are basically presented to analyse the performance of OFDM-FSO system in the weak regime and strong regime of the atmospheric turbulence modelling. The log-normal model represents the weak regime specifications in the OFDM-FSO system, it is a continuous distribution in the region of weak fluctuations, the statics of the irradiance fluctuations have experimentally found to obey the log-normal distribution [16]. The log intensity variance $\sigma_{1}^{2}=4 \sigma_{x}^{2}$ and the mean log intensity is $E[I]=2 E[X]$. which is valid for real valued Gaussian random variables then the mean log intensity can be given as :

$$
E[I]=-\frac{\sigma_{l}^{2}}{2}
$$

Whereas the negative exponential turbulence model verified the strong irradiance fluctuations, during the saturation regime the value of scintillation index S.I $\rightarrow 1$. This saturation regime is also called fully developed speckle regime, here the mean log intensity would be $E[I]=I_{0}$, where $I_{0}$ is the mean received irradiance. There are various standards has been used to simulate the OFDM-FSO system link parameters and their numerical values used for simulations are given in the table1: 
Table 1: OFDM-FSO Link Parameters

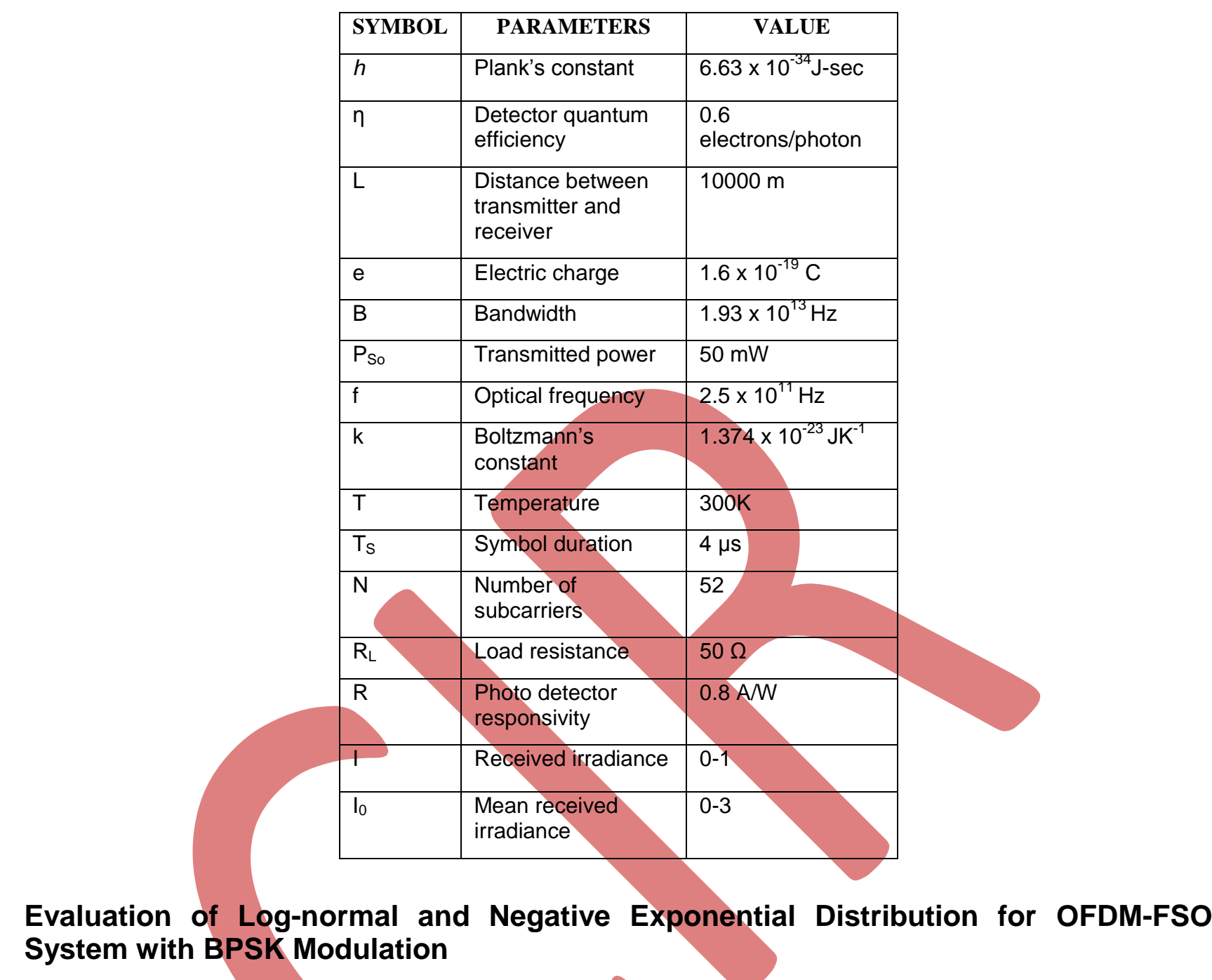

Binary Phase-shift keying (BPSK) is a digital modulation scheme that conveys data by changing or modulating, the phase of a reference signal (the carrier wave). BPSK is appropriate for low-cost passive transmitters and the BPSK is simplest form of phase shift keying (PSK). It uses two phases which are separated by $180^{\circ}$. The BER equation of BPSK modulation is as given below:

$$
B E R=\frac{1}{2} \operatorname{erf} c \sqrt{S N R}
$$

In this approach the FSO system is been modulated by the BPSK modulation technique, over this link OFDM system is applied. The comparison of Log-normal distribution and Negative Exponential distribution model is given in Fig. 2 and Fig. 3 respectively. The resultant curve is between BER and SNR, in Fig.2 which shows that at $7 \mathrm{~dB}$ of signal to noise ratio (SNR), the bit error rate(BER) of the system with OFDM gives $5 \times 10^{-3}$ for the Log-normal distribution. It couldn't be possible to reduce the received BER for the Log-normal distribution. If SNR value Further increased then there are no errors occurring in the received signal which means that BER becomes zero.

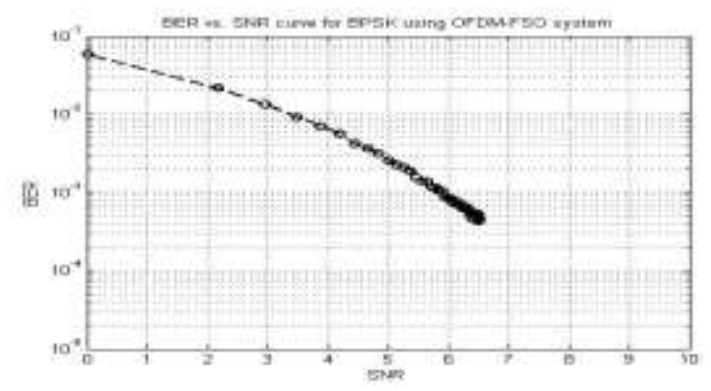

Fig. 2: BER vs. SNR curve for OFDM-FSO system for Log-normal Distribution with BPSK Modulation 
The curve given in Fig.3 shows the Negative Exponential Channel model modulated by BPSK modulation. The curve depicts that at $7 \mathrm{~dB}$ of signal to noise ratio (SNR), the bit error rate(BER) of the FSO system with OFDM gives $9 \times 10^{-3}$ for the current distribution. After the SNR value is increased above $9 \mathrm{~dB}$ then there are no errors occurring in the received signal.

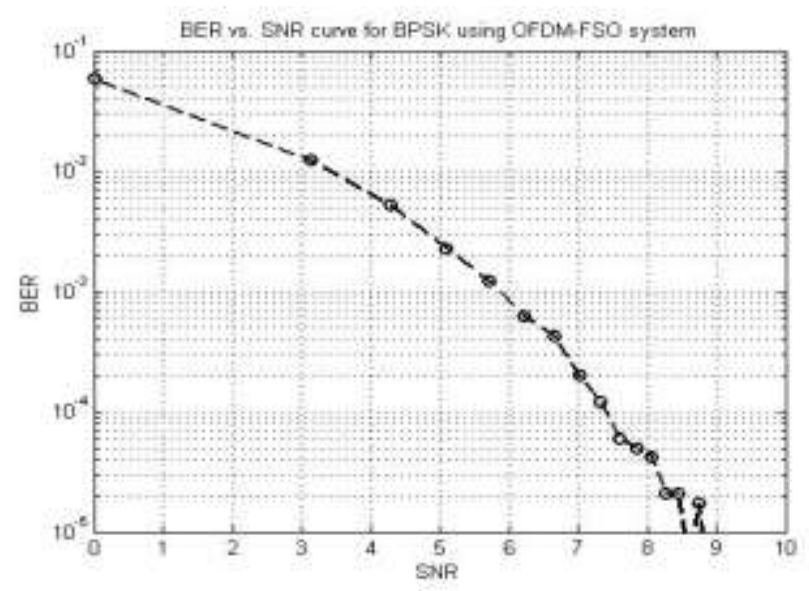

Fig. 3: BER vs. SNR curve for OFDM-FSO system for Negative Exponential Distribution with BPSK Modulation

If there is a comparisonbetween the Log-normal distribution and Negative Exponential Distribution over the same SNR value then from both the curves in Fig. 2 and Fig. 3 represents that data rate increases as per as the BER increases over the and same power for the Log-normal distribution, also analyses that OFDM technique is efficiently used with the FSO system, with BPSK modulation. The BPSK modulation has a disadvantage of wastage of the bandwidth as one symbol getting transmitted carries only one bit (i.e. 1Bit/symbol)

\section{Evaluation of Log-normal and Negative Exponential Distribution for OFDM-FSO System with QPSK Modulation}

The QPSK is can be used to double the data rate compared with a BPSK system while it maintains the same bandwidth of the signal. The QPSK can also work in a manner, in which it maintains the data-rate of BPSK but make the bandwidth requirement half as compared to BPSK. The bit error rate equation of QPSK modulation is given as:

$$
B E R=\operatorname{erfc} \sqrt{S N R}
$$

The performance of Log-normal and Negative Exponential distribution with QPSK modulation is given by Fig. 4 and Fig. 5 respectively,with OFDM QPSK-FSO modulation the BER isapproximate $3 \times 10^{-2}$ at SNR of $7 \mathrm{~dB}$ for the Log-normal distribution. There is no possibility to reduce such BER value at the given SNR value. The SNR cannot be further increased as there would be no BER received.

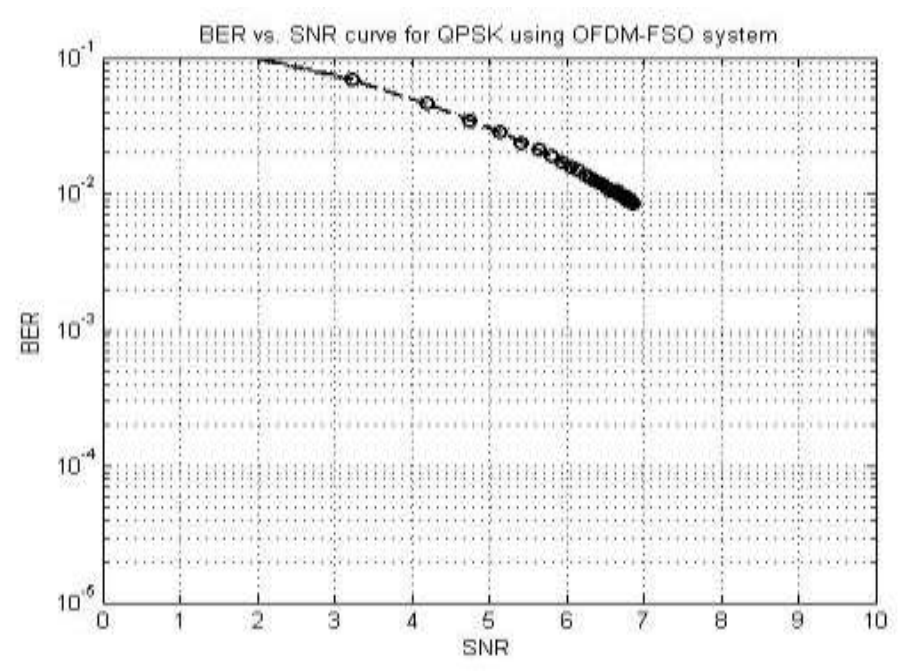


Fig. 4: BER vs. SNR curve for OFDM-FSO system for Log-normal Distribution with QPSK Modulation

The curve given in Fig. 5 is for the negative exponential model which shows that the BER is $6 \times 10^{-3}$ at the same SNR value of7 $\mathrm{dB}$. For the Negative Exponential distribution which is for the saturated strong regime at this value of SNR, the BER is achievable to this value only.

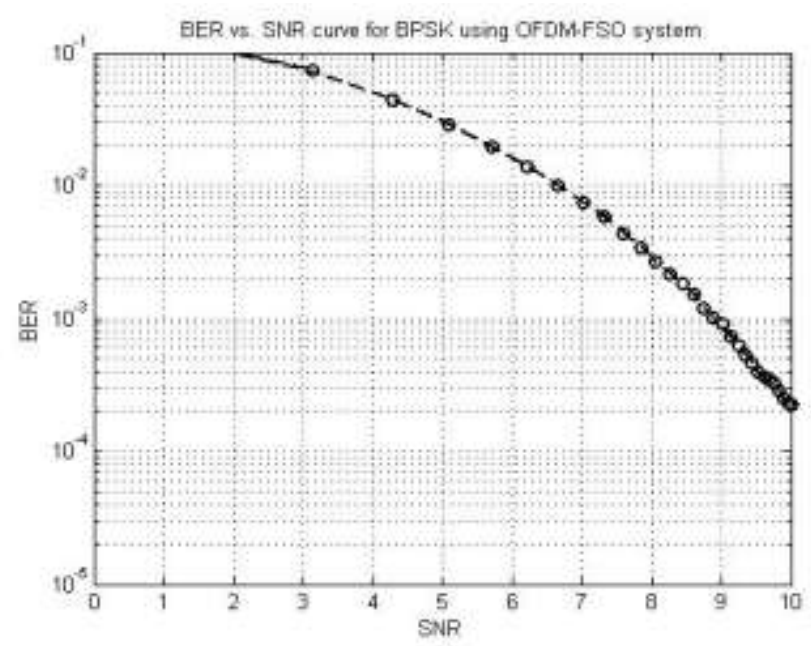

Fig. 5: BER vs. SNR curve for OFDM-FSO system for Negative Exponential Distribution with QPSK Modulation

From both the above results it is clear that for Log normal distribution has increased data rate as the result of bit error rate is analyzed. Fig. 4 and Fig. 5 shows the results of BER vs. SNR for QPSK modulated system with Log-normal distribution and Negative Exponential distribution respectively.

\section{Evaluation of Log-normal and Negative Exponential Distribution for OFDM-FSO System with 8-PSK Modulation}

In 8PSK modulator, every 3 input data bits are mapped to one complex-valued 8PSK symbol (where as in QPSK 2 input bits are mapped to one complex-valued QPSK symbol). Each symbol is gray coded prior to mapping them to the 8-PSK constellation (this is to allow only one bit change in the constellation, thereby avoiding abrupt phase changes in the constellation which may lead to addition errors in the receiver)[17] .the BER for 8-PSK modulation technique can be given as :

$$
B E R=2 Q \operatorname{erfc} \sqrt{S N R}
$$

The resultant curve of BER vs. SNR in case of 8-PSK modulation with OFDM system is given by Fig. 6 and Fig. 7. The curve for the Log-normal distribution in Fig. 6 shows that the BER received is $2 \times 10^{-1}$ at SNR of $7 \mathrm{~dB}$.

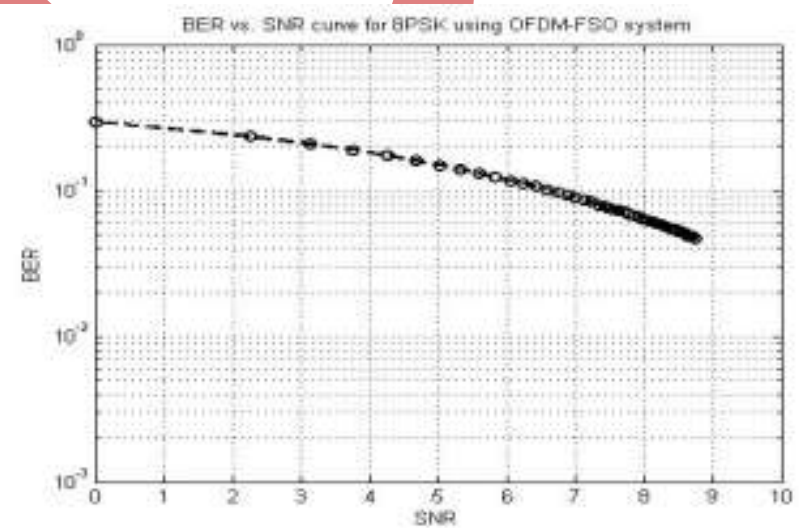

Fig. 6: BER vs. SNR curve for OFDM-FSO system for Log-normal Distribution with 8-PSK Modulation

The curve in Fig. 7 portrays the performance of the Negative Exponential model which shows that at $7 \mathrm{~dB}$ of SNR value the BER is near about $4 \times 10^{-1}$. 


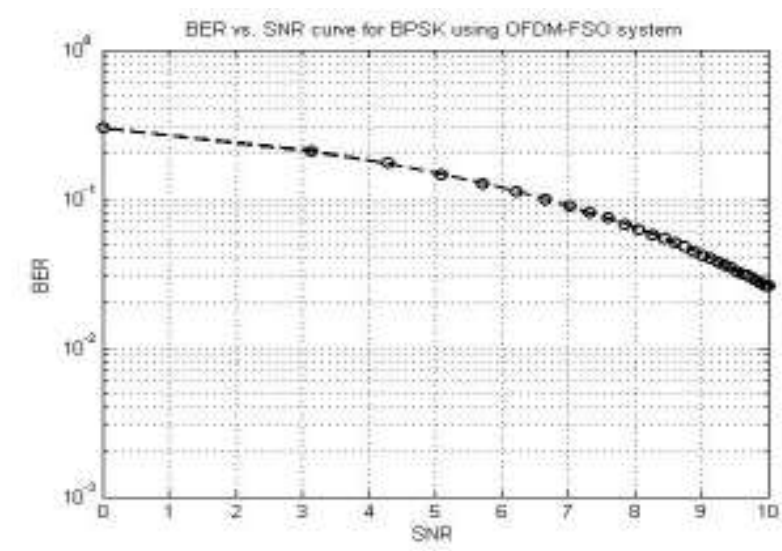

Fig. 7: BER vs. SNR curve for OFDM-FSO system for Negative Exponential Distribution with 8-PSK Modulation

For 8-PSK modulation technique it is not possible to achieve the bit error rate lesser than the received value at the same power adapted to the same SNR. From both the resultants it is predicted that the data rate for the Log normal model is superior than that for Negative exponential distribution.

\section{CONCLUSION}

The OFDM-FSO communication system integrates two developed technologies and gives better results for the several modulation techniques. This paper analysed the performance of Log-normal and Negative Exponential distribution for various modulation techniques. From the results it is clear that BPSK modulation provides much more BER improvement than all the other modulation schemes applied over both the distribution system. The distribution models Log-normal and Negative Exponential are of weak and strong turbulence regime respectively. In terms of BER improvement the Negative Exponential Model gives better results than log-normal distribution with each modulation technique. However, by using the BPSK modulation technique for the OFDM-FSO system Negative Exponential distribution gives better results than other modulation techniques. So, it is clear that Negative Exponential modeling is better than that of Log-normal distribution in terms of BER improvement with BPSK modulation over the OFDM-FSO system link.

\section{ACKNOWLEDGMENTS}

First of all I would like to thank Mr. Gurpartap Singh for his support to make this research paper complete and I would like to thank to my parents and friends for their all kind of help, without their help this work cannot be completed.

\section{REFERENCES}

[1] S. Bloom, E. Korevaar, J. Schuster, and H. Willebrand. 2003. "Understanding the performance of free-space optics," Journal of Optical Networking, Optical Society of America, Vol. 2, Issue 6, pp. 213-222.

[2] H. Willebrand and B. S. Ghuman. 2002."Free Space Optics: Enabling Optical Connectivity in Todays Network. Indianapolis", SAMS. Vol. 06, Issue. 05, pp. 43-48.

[3] X. Zhu and J. M. Kahn. 2002. "Free-space optical communication through atmospheric turbulence channels," IEEE Transactions on Communications, Vol 50, Issue. 07, pp. $1293-1300$.

[4] I. I. Kim, B. McArthur, and E. Korevaar. 2001."Comparison of laser beam propagation at $785 \mathrm{~nm}$ and $1550 \mathrm{~nm}$ in fog and haze for optical wireless communications," Proc. SPIE Opt. Wireless Commun. III, Vol. 14, Issue. 09, pp. 2637

[5] K. Wakafuji and T. Ohtsuki. 2005."Performance analysis of atmospheric optical subcarrier-multiplexing systems and atmospheric optical subcarrier Modulated code-division multiplexing systems," J. Lightw. Technol., Vol. 23, Issue. 04, pp. 16761682.

[6] A. Papoulis.1991."Probability, Random Variables, and Stochastic Processes," 3rd Edition, McGrawHill, New York, USA.

[7] M. Al Naboulsi, H. Sizun, and F. de Fornel. 2005. "Propagation of optical and infrared waves in the atmosphere," in Wave Propagation and RemoteSensing, Boulder, CO, USA.

[8] K. Tsukamoto, A. Hashimoto, Y. Aburakawa and M. Matsumoto. 2009. "The case for free space," IEEE Microw. Mag., Vol. 10, Issue. 05, pp. 84-92.

[9] S. Hranilovic and A. Mostafa. 2012. "In-Field Demonstration of OFDM-Over-FSO," IEEE Photonics Technology Letters, Vol. 24, Isuue. 08, pp. 132-137.

[10] S. Rosetti and G. E. Corazza. 2012. "OFDM Channel Estimation Based on Impulse Response Decimation: Analysis and Novel Algorithms," IEEE Transactions On Communications, Vol. 06, Issue. 07, pp. 326-367. 
[11] A. N. Kolomogrov. 1956. "On the Shannon theory of information transmission in the case of continuous signals," IRE Transactions on Information Theory, Vol. 2, Issue. 04, pp. 102-108..

[12] X. Zhu and J. Kahn. 2002."Free-space optical communication through atmospheric turbulence channels," IEEE Trans. Commun. Vol. 24, Issue. 05, pp. 1293-1330.

[13] Z. Ghassemlooy, W. Popoola, S. Rajbhandari. 2013. "Optical WirellessCommunication :Syatem and channel Modelling with Matlab". CRC Press, USA.

[14] S.G. Wilson. 1996. "Digital Modulation and Coding,'New Jersey Press, Prentice Hall, USA.

[15] L. C. Andrews. 2004. "Field Guide to Atmospheric Optics," The Society of Photo-Optical Instrumentation Engineers. Vol. 02, Issue. 01, pp. 59-66.

[16] T.D. Rappaport. 2009. “Wireless Communication Principles and Practice,"Second edition, New Delhi, Pearson Education India.

[17] F. Xiong. 2006. "Digital Modulation Techniques, “(Second edition), Norwood, MA, Artech House, Inc., USA.
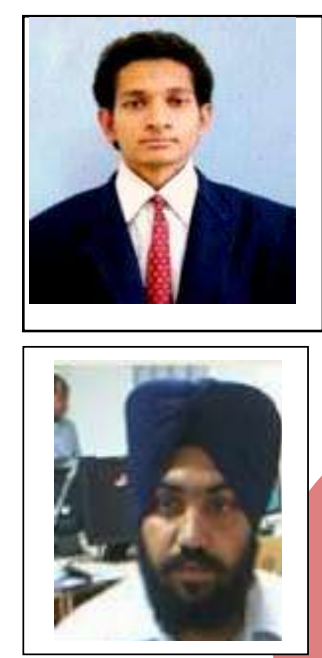

He was born in Jhansi, Uttar Pradesh (India). He has completed his bachelor in technology degree in ECE, from Pranveer Singh Institute of Technology. Currently he is pursuing his M.tech-ECE from Lovely Profesional University, INDIA. His area of interest is Wireless Optical Communication.

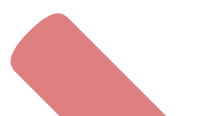

He was born in Tarn taran, Punjab (India). He has completed his B.tech-ECE and M.tech-ECE from Lovely Professional University, India. Currently he is working as an assistant professor in Lovely Professional University, Phagwara, India. He has published numerous journals in various International Journals. His area of research is Wireless Optical Communication 